\title{
Using a choice experiment to estimate the benefits of a reduction of externalities in urban areas with special focus on electrosmog
}

\author{
Running title: Willingness to pay for improved environmental \\ quality \\ Dr. Silvia BANFI* \\ Prof. Dr. Massimo FILIPPINI*^ \\ Andrea HOREHÁJOVÁ**
}

\begin{abstract}
Traffic noise, air pollution and electromagnetic pollution (i.e. non-ionizing radiation, also called electrosmog) are typical negative local externalities in urban areas. They are side-effects of human and economic activity (e.g. road transport, telecommunication) and they affect individuals' well-being negatively without compensation. In recent years, the increased number of mobile phone antennas in residential areas, and thus the increased intensity of radiated power, has aroused public concern, discussions and protests. The view of an antenna is annoying an increasing number of inhabitants. In this paper, the stated Choice Experiment (CE) is used to estimate the Willingness To Pay (WTP) residents in the cities of Zurich and Lugano place on the reduction of these three environmental loads. Estimation results reveal that there is a positive and significant WTP for a reduction of air pollution and traffic noise levels to those limit values fixed by the government. Respondents also show WTP for reducing electrosmog and removing mobile phone antennas from their view, however to a lesser extent. In addition, this is the first study that estimates the benefit of a reduction of electrosmog using a CE.
\end{abstract}

Key words: choice experiment, electrosmog, traffic noise, air pollution. JEL classification: C25, C93, Q51, R21

\footnotetext{
* Swiss Federal Institute of Technology, Centre for Energy Policy and Economics (CEPE), Zurich, Switzerland

- Università della Svizzera italiana, Istituto di microeconomia e economia Pubblica, Lugano, Switzerland

• Corresponding author: ahorehajova@ethz.ch
} 


\section{Introduction}

Electromagnetic pollution (i.e. non-ionizing radiation, also called electrosmog1), air pollution and noise are typical negative local externalities in urban areas. They are side-effects generated by human and economic activity (e.g. road transport, telecommunication) and they affect individuals' well-being negatively without compensation.

Numerous mobile phone antennas have been installed in Switzerland from 1998, with a sharp rise in the five years from 2000 to 2005, and it is expected that their number will further increase. ${ }^{2}$ The rising number of mobile phone antennas in residential areas, and thus the increased intensity of radiated power, has as well, in the recent years, aroused public concern, discussions and protests. On the other hand, measurement of the electrosmog ${ }^{3}$ caused by mobile phone antennas in the Swiss cities showed that in general the radiations are within the level prescribed by law and epidemiological studies have not made conclusive assessments about the potential negative health effects of electrosmog exposure. ${ }^{4}$ Despite the lack of information on electrosmog and the uncertainty of its impacts on health, most people are concerned about the increasing intensity of radiated power in inhabited areas. For instance, studies performed by Siegrist et al. $(2003,2005)$ show that people viewed risks associated with cell phones or mobile phone antennas as high.

Measurements carried out in 2005 and 2006 show that in several Swiss cities the limit values of air pollution fixed in the Swiss law have often been exceeded. Moreover, in several areas of these cities also, the day and night standards for the noise level were violated.

In order to solve these environmental problems, policy makers are evaluating the possibility of introducing new environmental instruments to improve the quality of the environment in the Swiss cities. This paper aims at helping policy makers to

\footnotetext{
1 Electromagnetic pollution caused mainly by mobile phone antennas_(also known as "base stations"), TV and radio transmitters and high voltage power lines. In this study we do not consider electrosmog emitted inside a dwelling or house, since this kind of pollution can not be considered as an externality for the household.

2 This fast expansion of the mobile network was caused by the rapid growth in the mobile telecommunication.

3 In terms of an application of the precautionary principle, the Swiss federal state and the mobile phone companies agreed in 2006 to enforce the controls of the radiation levels of the antennas.

${ }^{4}$ For a review of these studies see Ahlbom et al. (2001) and Breckenkamp et al. (2003).
} 
evaluate different environmental policies by focusing on the estimation of the individual Willingness To Pay (WTP) associated with the reduction of electrosmog, ${ }^{5}$ levels of air pollution and noise to the levels stipulated in the Swiss standards. Indeed, when policy makers have to decide on introducing new environmental policy measures, the costs of these are often known since market prices are observable. ${ }^{6}$ For example, an environmental policy targeted at reducing electromagnetic radiation of mobile phone antennas could imply additional costs for the operators because of the need to install more antennas in order to provide the same reception in a specific area. While costs can be estimated by using such market data, social benefit measures are more difficult to obtain. The information provided by this article on the potential benefits of an improvement in urban environmental quality can be used later in a cost-benefit analysis.

For the estimation of the economic benefits of a reduction of the electrosmog, levels of air pollution and noise, a stated Choice Experiment (CE) was used. The CE has its background in Lancaster's attribute theory of consumer choice (Lancaster, 1966) and in the Random Utility Theory (RUT, McFadden, 1974; Manski and Lerman, 1977). Only in recent years it has been used for the valuation of environmental attributes. ${ }^{7}$

However, we are not aware of any studies valuing the economic impacts of the electrosmog that make use of CE approach. Generally, the empirical literature on the economic impacts of the sources of electromagnetic fields (e.g. mobile phones, base stations, high-voltage transmission lines) is poor. There are only few empirical studies for the US, Canada and Switzerland that have examined the impact of the presence of electromagnetic fields on the rents for dwellings using the marketbased hedonic model. ${ }^{8}$ Banfi et al. (2007) have estimated hedonic price functions using revealed data for the Swiss cities of Zurich and Lugano. The main findings show a significant negative impact of electrosmog, air pollution and traffic noise on

\footnotetext{
${ }^{5}$ As a proxy for electrosmog we used the presence of a mobile phone antenna within 150 meters of a dwelling.

${ }^{6}$ For examples of cost-benefit analysis, of measures aiming at improving air quality where costs of the measures are known, see Olsthoorn et al. (1999) or Harford (2006).

7 For a discussion of the application of CEs to value the environment, see Hanley et al. (1998).

8 For instance Hamilton and Schwann (1995) focus on the impact of high-voltage transmission lines on the sale values of houses in the Vancouver area. Des Rosiers (2002) investigated the impact of highvoltage transmission lines on surrounding property values in the City of Brossard.
} 
the rents for dwellings. For example, the presence of an antenna less than 200 meters from a residential building decreases rents by around $2 \%$.

In the context of environmental pollution, several CE studies have been conducted of air quality valuations (Ortuzar and Rodrıguez, 2002) and noise valuations (Garrod and Willis, 1999; Galilea and Ortuzar, 2005). There are only few empirical studies that have examined both air quality and noise valuations (Sælensminde, 1999; Wardman and Bristow, 2004).

Therefore, there are at least two novel aspects of this research. First, this article estimates the WTP for a reduction of electrosmog with the help of a CE. Second, in the same study we have examined the WTP for electrosmog, air quality and traffic noise reduction. This allows us to compare the WTP for the improvements of different environmental characteristics.

There are various methods for the valuation of environmental amenities. These differ greatly in their data requirements, in their assumptions and also in the types of benefits they are able to measure. Broadly, these methods can be divided into two groups; revealed and stated preference approaches, or in other words, approaches based on real or hypothetical markets. The decision to use a $\mathrm{CE}$, as one of the stated preference methods, was motivated by the hypothetical nature of quality change (a non-existent situation, e.g. the reduction of threshold levels for radiation of a mobile phone antenna) we wanted to evaluate. Moreover, the $\mathrm{CE}$ determines the values for each possible environmental outcome of the experiment. The other stated preference method, Contingent Valuation Method (CVM), usually provides a single value for an expected quality change. Further, the $C E$ is more efficient in terms of data collection than the CVM. With the same number of respondents, the CE provides the higher number of useful observations because each respondent is faced with sets of multi-attribute alternatives.

The rest of the article proceeds as follows: Section 2 presents the model specification used in this article. The experiment design and the data are described in Section 3 and 4. Section 5 presents the estimation results and discusses their implications. Finally, the main conclusions are summarized in the Section 6.

\section{Model specification}

In this article, we employed the RUT to model an individual's choice among a choice set of dwellings composed by the actual choice and several hypothetical alternative 
choices. The RUT has already been used in the literature to value environmental attributes of housing. ${ }^{9}$ In this framework, given a finite set of alternative dwellings characterized by distinct environmental attributes, the individual $n$ chooses the dwelling $i$ that yields the highest utility. According to the RUT, the utility of goods or services, in our case dwellings, is the sum of a deterministic component, $V_{i n}$, and a random component, $\varepsilon_{\text {in. }}$. Therefore, the general model can be specified as a stochastic conditional (conditional on the choice made) indirect utility function of the form

$$
U_{\text {in }}=V_{\text {in }}\left(y_{n}-P_{i}, Z_{i}, C_{n} ; \beta\right)+\varepsilon_{\text {in }} \quad i=1,2,3, \ldots . I \quad n=1,2,3, \ldots . N
$$

where $y_{n}$ is income of household $n, P_{i}$ the price paid for the dwelling choice option $i$, $Z_{i}$ a vector of observed dwelling attributes, $C_{n}$ a vector of observed individual characteristics and $\beta$ a parameter vector.

The probability $P_{n}(i)$ that individual $n$ chooses dwelling $i$ rather then dwelling $j$ is

$$
P_{n}(i)=P\left(V_{i n}\left(y_{n}-P_{i}, Z_{i}, C_{n} ; \beta\right)+\varepsilon_{i n} \geq V_{j n}\left(y_{n}-P_{j}, Z_{j}, C_{n} ; \beta\right)+\varepsilon_{j n}\right)
$$

Assuming that the random component follows an independent and identically distributed (i.i.d.) extreme value type I distribution, the probability $P_{n}(i)$ that individual $n$ chooses dwelling $i$ can be written in a logit model of the following form:

$$
P_{n}(i)=\frac{\exp \left(\mu V_{i n}\right)}{\sum_{j=1}^{I} \exp \left(\mu V_{j n}\right)}
$$

where $I$ is the number of dwelling choice options, and $\mu$ a scale parameter, which is usually assumed to be equal to one. Expression (3) is the basic equation of a multinomial/conditional logit (cf. Greene, 2003).

In our CE, we used a conditional logit model; this means we assume that the values of the choice characteristics vary across choices, while the parameters are common across the choices. In this case, the social and economic characteristics of the households are constant across choices for any given household; they can only enter the model as interaction terms with the dwelling attributes.

\footnotetext{
9 See, for instance, the studies by Earnhard (2001) and Chattopadhyay et al. (2005). Other examples are studies by de Blaeij et al. (2007) and Shen et al. (2008).
} 
The conditional indirect utility function,$V_{i n}$, considered in this study is assumed to be linear in parameters.

$$
V_{i n}=\beta_{0}+\beta_{y}\left(y_{n}-P_{i}\right)+\beta_{1} Z_{1}+\beta_{2} Z_{2}+\ldots \ldots+\beta_{h} Z_{h}
$$

The conditional logit model is estimated using the maximum likelihood estimation method. Once the model parameters are estimated and assuming constant marginal utility of income, a welfare measure can be calculated. For instance, for a household $n$ facing a choice set $/$ the expected Compensating Variation (CV) can be computed using the following expression:

$E(C V)=\frac{\ln \sum_{i} \exp \left(V_{i 1}\right)-\ln \sum_{i} \exp \left(V_{i 0}\right)}{\gamma}$

where $\gamma$ represents the constant marginal utility of incomes, and $V_{i 1}$ and $V_{i 0}$ the indirect utility functions after and before the change considered in the CE. Moreover, the estimated coefficients can be used to estimate the marginal price of each attribute which, assuming short-run equilibrium in the housing market, is equal the marginal WTP for that attribute. The marginal WTP for a change in a single attribute can be represented as a ratio of the coefficients:

$$
M W T P=-1\left(\frac{\beta_{\text {attribute }}}{\beta_{\text {monetary variable }}}\right)
$$

\section{Experiment Design}

In order to examine the impact of these three externalities on the rent for dwellings, we have conducted a CE in the cities of Zurich and Lugano. The choice of these two cities $^{10}$ is mainly motivated by the fact that these cities are highly affected by air pollution and traffic noise. Moreover, Zurich is located in the German part of Switzerland, whereas Lugano is located in the Italian part. This allows the identification of potential differences in the evaluation of environmental improvements across different cultures.

\footnotetext{
10 One of the differences between these two cities is their size. While Zurich is the largest city in Switzerland with more than 370 '000 inhabitants, Lugano is one of the average cities regarding the number of inhabitants (around 26'000).
} 
The dataset used for this CE comprises a sample of 394 households for Zurich and 241 households for Lugano. In the $C E$, each respondent faced six choice sets. In each choice set respondents were asked to choose between three alternatives. To reduce the hypothetical character of the $\mathrm{CE}$, the third alternative always indicated the current dwelling situation of the respondents. This third option of choosing none of hypothetical alternatives, commonly called status quo, stated that there would be no changes in the environmental attributes of the dwelling. Alternatives 1 and 2 were characterized by a change in the rent and in the environmental attributes of the dwelling with respect to the status quo alternative.

Based on the discussions with the representatives of the Swiss Federal Office for the Environment (FOEN), a monetary attribute, the monthly rent and four environmental attributes were chosen: view of a mobile phone antenna, presence of a mobile phone antenna within 150 meters from the dwelling, air quality and traffic noise exposure. The levels of these attributes were defined as follows:

- Monthly rent: the monthly rent was related to the current rent for the apartment. According to the change (improvement or deterioration) in the environmental attributes of the dwelling the rent was varied by $+10 \%,+7 \%$, $+5 \%,+2 \%$ or $-2 \%,-5 \%,-7 \%$ and $-10 \% .{ }^{11}$ The choice situation, however, was presented with a newly computed rent (the rent indicated by the respondent and the percentage change).

- View of mobile phone antenna: two levels were defined; yes and no.

- Mobile phone antenna in the surrounding (150m): this attribute had 3 different levels: 1) no antenna in the surrounding, 2) antenna in the surrounding with a 10 times lower limit value than the one fixed in the safety guidelines of the government; and 3) antenna in the surrounding that does not exceed the limit values of the safety guidelines.

- $\quad$ Air quality: the air quality represents an overall air quality and was defined with three levels; good, medium and bad. Good air quality was defined as the situation where values of air quality clearly fall below the limit values; medium as the situation where the limit values are just preserved and low as the situation when the limit values are clearly exceeded.

\footnotetext{
11 The percentage changes were selected after broad literature review, see, for example, Sælensminde (1999); Ortuzar and Rodriquez (2002) and Wardman and Bristow (2004).
} 
- $\quad$ Traffic noise exposure: for this attribute also three levels were defined. Low traffic noise exposure as in quiet small streets; medium as in streets with moderate traffic and high as on a highway or on a road with heavy truck traffic. This latter level implies that the limit of the noise imposed by the law is exceeded.

Table 1

A typical choice screen presented to the respondents is illustrated in Table 1. Respondents were asked to imagine their current dwelling situation would change with regard to the above-mentioned attributes, with all other dwelling characteristics such as number and size of rooms, interior, floor etc. remaining the same. Then they were asked to select out of the three alternatives the one most preferred. Respondents were provided with the description of the different attributes and their levels in the form of pop-up windows. ${ }^{12}$

Given the five attributes and their associated levels, 432 potential profiles ${ }^{13}$ exist. A fractional factorial design was used to combine the attribute differences. We reduced the number of profiles to 24 . Depending on the participant's current dwelling situation, 12 profiles have been randomly selected for CE by the computer program used to administer the CE. We utilized a web-based survey and we proceeded in two steps. In the first step, a random sample of potential participants ${ }^{14}$ selected from the telephone directory were contacted by phone and asked if they were interested to participate in the survey. In the second step we sent an e-mail with an official invitation to participate in our survey and with a link and password to fill in the questionnaire.

\section{Data description}

The survey was conducted during the summer 2005 and consisted of three parts. The first part collected information about the dwellings' characteristics and surrounding environment quality such as the traffic noise exposure, air quality and presence of mobile phone antennas in the neighborhood. The CE was the centre of the questionnaire, and the last part contained questions regarding the participant's socio-economic status, such as age, education etc. and household income. The

\footnotetext{
${ }^{12}$ Further details of the choice experiment can be found in the book by Banfi et al. (2007).

$133^{3} \times 2 \times 8$

14 The sample consisted of inhabitants living in rented dwellings in Zurich and Lugano for at least 12 months.
} 
questionnaire and the attributes used in the CE were developed after discussions with researchers specialized in electrosmog, noise and air quality, and in-depth literature review.

The original data sets collected from the second step consist of 409 participants from Zurich and 258 from Lugano, corresponding to the response rate of $72 \%$ and $66 \%$ respectively. This sample is further reduced by omitting a number of observations because of missing data or inconsistent responses. After removing such observations, the final regression sample was reduced to 394 participants (2'364 choice situations) for Zurich and 241 (or 1'442 choice situations ${ }^{15}$ ) for Lugano. The descriptive summary of this sample is presented in Table 2. The upper part of the table lists the socio-economic characteristics of the respondents; the middle part states the attributes of their current dwellings; and the lower part lists the attributes of the alternatives offered in the CE.

There are several characteristics of the participants that we can see directly from Table 2. The gender distribution of our sample with $47 \%$ of males in Zurich and $49 \%$ in Lugano is similar to the cities' averages. ${ }^{16}$ The ages ranged from 19-85 years in Zurich and 19-95 years in Lugano, with an average age of 40 in both cities.

Among the participants, $42 \%$ in Zurich and $44 \%$ in Lugano had a university education, and $29 \%$ and $23 \%$ respectively had a vocational training/ apprenticeship. The shares of respondents with vocational qualifications follow the official statistics for the both cities (Census 2000). However, the official statistics indicate lower shares for people with a university degree, 23\% and 20\% respectively. The respondents with university education are therefore overrepresented. There are two possible reasons that can explain this sample characteristic. First, the respondents with university education tend to be more interested in the discussion and solution of environmental problems, as shown for Switzerland by Diekmann and Meyer (2008). Second, the choice of the two official languages, German and Italian, excluded automatically the foreign people not fluent in one official language. As shown in the cantonal statistics on education, a large proportion of foreign people have just a basic education. This has to be considered by the interpretation of the empirical results.

The participants' average household income was between 5'000 and 6'000 Swiss Francs (CHF) for Zurich and for Lugano between 6'000-7'000 CHF, with a Standard

\footnotetext{
${ }^{15}$ Computed as, number of respondents times the number of choice cards. In the case of Lugano there were four missing choice situations.

${ }^{16}$ Statistics of the city of Zurich (2005); and Federal Office of Statistics, population statistics (2003).
} 
Deviation (SD) between 2'000 and 3'000 CHF for both cities. The median income lay between 5'000 and 6'000 CHF. These values are comparable to the values presented in the Swiss income survey (2004). For instance, the median income for canton Zurich is around 6'000 CHF and for canton Lugano 5'000 CHF. Unfortunately, no detailed statistics are available for the two cities.

Regarding the environmental characteristics of the current dwellings the sample can be described as follows: around $40 \%$ of the participants in Zurich and $42 \%$ in Lugano perceive their air quality as bad; one fifth in Zurich and one fourth in Lugano think that the air quality of their current dwellings is good. Concerning the traffic noise exposure, the share of participants with high traffic noise exposure is $31 \%$ in Zurich and 25\% in Lugano. Almost half of the participants from Lugano think their traffic noise exposure is low. This share is lower in Zurich with $36 \%$ of the participants. For $31 \%$ of the apartments in Zurich there is a mobile phone antenna with present limit values within $150 \mathrm{~m}$; in Lugano this share is slightly lower with $26 \%$ of the apartments.

The average monthly rent is 1'585 CHF in Zurich and slightly lower in Lugano, with 1 '442 CHF. The median monthly rent is 1'485 CHF and 1'400 CHF respectively.

Each participant decided for one alternative in each of six choice situations. The share of participants who always preferred their current dwelling situations over other alternatives is $20 \%$ in Zurich and $22 \%$ in Lugano. These shares are not so large comparing to the shares from other studies. ${ }^{17}$

The lower part of Table 2 gives a descriptive summary of the characteristics of the hypothetical offers. These can be described as a balanced sample in that there is a comparable share of apartments with good, medium and bad air quality in the offered alternatives. This applies also to traffic noise exposure and presence of a mobile phone antenna. The monthly rent of alternatives varies from 450 to 7'056 CHF in Zurich and from 450 to 3'920 CHF in Lugano, with an average of 1'556 CHF and 1 ' 410 respectively. In both samples the average monthly rent of the alternatives is about the same as the average monthly rent of the current dwelling situations.

Table 2

17 See Banfi et al. (2008) 


\section{Estimation results}

The CE data were analyzed using the conditional logit model. The explanatory variables included in the estimation are: the monthly rent for the dwelling; two dummy variables for presence of a mobile-phone antenna, air quality and traffic noise exposure with the worst level being chosen as the reference category (presence of a mobile phone antenna with present limit values, bad air quality and high traffic noise exposure). Further, we introduced a dummy variable for the view of an antenna and a dummy variable that takes value one for the status quo and zero for the two hypothetical alternatives that imply changes in the environmental attributes of the dwelling.

The two cities are considered to be separate markets ${ }^{18}$ and thus the data were evaluated and estimated individually. We estimated for each sample of the two cities, two models: the basic model and the extended model. Both models include all the experimental design variables and the alternative-specific constant. In addition, the extended model includes a number of individual characteristics through interaction terms. The variables considered for interaction terms are: household income (interacted with the rent), family members with allergies (interacted with air quality) and education level (interacted with the presence of a mobile phone antenna). ${ }^{19}$ Besides these classical socio-economic characteristics, we considered in the estimation of the extended models both a dummy variable that takes value one, if the rent of the alternative dwelling choices was lower than the rent for the status quo (otherwise zero), and an interaction variable between monthly rent and frequency of choosing the status quo. ${ }^{20}$

The results of the estimations for both models are presented in Tables 3 and 4 .

Table 3

\footnotetext{
18 This assumption is based on the results of two studies, Banfi et al. (2007) and Diekmann and Meyer (2008), confirming the differences in perceptions and attitudes towards the environmental loads in these two cities.

${ }^{19}$ The interaction between the education level and presence of a mobile phone antenna was added to the extended model based on the discussions with the researchers. In a subsequent study by Diekmann and Meyer (2008) the authors found a negative correlation between the educational level and perceptions of risks from mobile phone antennas.

${ }^{20}$ Further interaction terms were tested but, since not significant and theoretically not necessary, they were excluded from the extended model.
} 


\section{Table 4}

The interpretation of the coefficient values is not straightforward, except for the significance and relative size. All experimental design attributes are significantly different from zero at $1 \%$ significance level and have the expected sign in both models and for both samples.

The coefficient of the dummy variable for the view of the antenna is negative. This implies that households tend to not choose a dwelling with this characteristic. As expected, the coefficient of the monthly rent is negative. All other experimental design attributes have a positive coefficient estimate. This means that improving the environmental characteristics of a dwelling will increase its probability to be chosen. Furthermore, from the magnitude of the coefficients one can see that participants are more likely to prefer the better attribute level to the worse attribute level. For example, starting from a situation with an antenna within 150 meters from the dwelling they prefer a situation without an antenna rather than a situation with an antenna in the surrounding with a 10 times lower limit value.

The alternative-specific constant is positive and significant. This result indicates that participants are averse to choosing hypothetical alternative dwelling situations for reasons that are not considered in the model.

In the extended model only few coefficients of the interaction variables are significant and have the expected sign. As expected, the interaction term between bad air quality and allergies is significant in both extended models. This result tells us that households whose members suffer from allergies are less likely to choose apartments with bad air quality.

The university education interacted with the nonpresence of an antenna shows that university educated people are more likely to choose an apartment with an antenna in the surrounding of 150 meters. However, this interaction term is not significant for the city of Lugano.

The significant coefficient of the interaction term between rent and high income level indicates that households with higher income (above 5'000 CHF per month) are more likely to choose more expensive dwellings in comparison to households with a medium income level (between 5'000 and 6'000 CHF per month). We could not observe a similar effect (with a negative sign) on the choices of low income households.

The interaction term between rent and the frequency of choosing the current dwelling situation is positive and significant. The environmental quality of more expensive flats is usually better; therefore, households with such conditions are less 
likely to choose an alternative. Finally, the variable indicating if the alternative is characterized by a lower rent than that for the current dwelling situation is not significant.

In a second phase using equation (6) and the results obtained in the extended model we calculated the average WTP for a change in the attributes. We chose to use the results of Model 2 because this model has a higher explanatory power than Model 1. The WTP (or implicit prices) for both samples are presented in Table 5.

\section{Table 5}

From Table 5, we gather that WTP is highest for a reduction of noise exposure from a level clearly above the limit to a level below the limit. Moreover, WTP for a reduction of the air pollution from a situation where the limit imposed by law is exceeded to a situation where this limit is clearly complied is also high. The slightly higher WTP for the reduction of traffic noise could be explained by its direct and immediate impact on well-being in comparison to the lagged effect of air pollution on people's health. The implicit prices for the avoidance of a mobile phone antenna in the neighborhood as well as for the presence of an antenna with stronger radiation limits are low. This is not surprising, since there is still no empirical evidence that electromagnetic radiation affects health. The WTP could be interpreted as a measure of precaution in order to avoid any risks coming from antennas. Further, some people can be considered as electromagnetic- sensitive (about $5 \%$ of population ${ }^{21}$ ); it can be expected that these persons have a higher WTP for a decrease in radiation. Finally, the WTP for avoiding the view of an antenna is lower than the WTP for avoiding the presence of an antenna. At any rate, this WTP of nearly $30 \mathrm{CHF}$ per month is not negligible.

Comparing the two cities, it is important to note that the WTP for reduction in noise and air pollution is greater in Zurich, whereas it is higher in Lugano for measures against electrosmog. This difference in the WTP could be due to cultural differences and differences in environmental awareness of the households. A study by Diekmann and Meyer (2008) on the environmental awareness of Swiss population shows that the perceptions and attitudes toward the environmental problems differ between households in the Italian and German parts of Switzerland.22 For example,

${ }^{21}$ Röösli et al.(2005)

22 These differences were also found in the study by Banfi et al. (2007). For example, traffic noise is the most irritating source of noise stated by the inhabitants of the two cities. $31 \%$ of respondents in Zurich and $25 \%$ in Lugano indicated they felt very annoyed by the traffic noise. 
people living in the Italian part are more concerned about the risks of radiation from mobile phone antennas, mobile phones and high voltage transmission lines. This could explain the higher WTP for removing a mobile phone antenna in the city of Lugano.

Looking at the 95\%-significance intervals, it can be recognized that the average WTPs have to be considered and treated with caution, since they are situated within a large interval.

These results are consistent with previous studies showing that households associate improved environmental quality with a reduced health risk and may choose to reduce the risk by moving from bad environmental conditions to dwellings with better environmental qualities. ${ }^{23}$

\section{Conclusions}

This article attempts to estimate the benefits of an increase in local environmental quality in two Swiss cities, Zurich and Lugano. Individuals' WTPs are estimated through a web-based CE, in which participants were asked to choose between their current and two different dwelling alternatives with varying environmental characteristics and monthly rent. The environmental characteristics considered are: air quality, traffic noise level, view of a mobile phone antenna and presence of such an antenna in the surroundings (until 150 meters).

This analysis contributes to the wide literature on environmental valuation studies by applying a stated preference approach to a new environmental field, that is the presence of mobile phone antennas in urban areas and in particular to the externalities due to radiation and impairment of view. The importance of this topic may increase in the next years with an increment of the number of mobile phone antennas. Further, the paper gives to policy makers (see Table 5) important information about the benefits of an improvement of air quality and a reduction of noise level to the limits set by law. In a second step, this information can be compared to the costs of policy measures suitable for reducing the pollution level under the allowed threshold value.

The estimation results show that not only the levels of traffic noise and air pollution are important when choosing a dwelling, but also the presence of mobile phone

23 These results were also confirmed by the hedonic study by Banfi et al. (2007). However, the comparison of the results has to be done with caution. 
antennas and the view on them play a role in this choice. Second, people show a positive and significant WTP for an improvement of environmental quality in the two urban areas. Low traffic noise exposure and good air quality are the highest valued attributes, while the presence and view of a mobile phone antenna shows a smaller WTP. Nonetheless, the magnitude of WTP for these last two effects is not negligible. In general, we can observe some differences in the magnitude of WTP between the two cities analysed.

Finally, it is important to mention also some limitations of this study: the $95 \%$ significance level of the WTP is quite broad. The use of the average WTP for policy purposes therefore needs particular caution. Other limitations are related to the design of the choice experiment: the increase or decrease in the rent chosen affects the WTP. Further, households with well-educated members are overrepresented in the samples, and there is a considerable share of respondents always choosing the status quo. We cannot exclude that these factors lead to some bias in the estimation results.

\section{References}

Ahlbom, A., Cardis, E., Green, A., Linet, M., Savitz, D. and Swerdlow, A. (2001) Review of the epidemiologic literature on EMF and health, Environmental Health Perspectives, 109 (Supplement 6), 911-933.

Banfi, S., Filippini, M., Horehájová, A. and Pióro, D. (2007), Zahlungsbereitschaft für eine verbesserte Umweltqualität am Wohnort. Schätzungen für die Städte Zürich and Lugano für die Bereiche Elektrosmog von Mobilfunkantennen, Luftverschmutzung und Lärmbelastung, vdf Hochschulverlag.

Banfi, S., Farsi, M., Filippini, M. and Jakob, M. (2006), Willingness to pay for energysaving measures in residential buildings, Energy Economics (forthcoming).

Breckenkamp, J., Berg, G. and Blettner, M. (2003), Biological effects on human health due to radiofrequency/ microwave exposure: A synopsis of cohort studies, Radiation and Environmental Biophysics, 42, 141-154.

Chattopadhyay, S., Braden, J.B. and Patunru, A. (2005), Benefits of hazardous waste cleanup: new evidence from survey- and market-based property value approaches, Contemporary Economic Policy, 23 (3), 357-375. 
de Blaeij, Arianne T., Nunes, Paulo A. L. D. and Bergh, Jeroen C. J. M. Van den (2007), 'Non-choice' options within a nested logit model: one model is insufficient, Applied Economics, 39 (10), 1245-1252.

Des Rosiers, F. (2002), Power Lines, Visual Encumbrance and House Values: A Microspatial Approach to Impact Measurement, Journal of Real Estate Research, 23 (3), 275-301.

Diekmann, A. and Meyer, R. (2008), Klimawandel, ökologische Risiken und Umweltbewusstsein in der Schweizerischen Bevölkerung, ETH-Studie Schweizer Umweltsurvey 2007.

Earnhard, D. (2001), Combining Revealed and Stated Preference Methods to Value Environmental Amenities at Residential Locations, Land Economics, 77 (1), 1229.

Galilea, P. and Ortuzar, J. de Dios (2005), Valuing Noise Level Reductions in a Residential Location Context, Transport Research: Part D, 10, 305-322.

Garrod, G. and Willis, K. G. (1999), Economic valuation of the environment: methods and case studies, Cheltenham: Edward Elgar.

Greene, W. H. (2003), Econometric Analysis, $5^{\text {th }}$ edition, Prentice-Hall International, UK.

Hamilton, S. W. and Schwann, G. M. (1995), Do High Voltage Electric Transmission Lines Affect Property Value?, Land Economics, 71 (4), 436-44.

Hanley, N., Wright, R. E. and Adamowicz, V. (1998), Using Choice Experiments to Value the Environment, Environmental and Resource Economics, 11 (3-4), 413428.

Harford, J.D. (2006), Congestion, pollution, and benefit-to-cost ratios of US public transit systems, Transportation Research Part D 11: 45-58.

Lancaster, K. (1966), A new approach to consumer theory, Journal of Political Economics, 74, 217-231.

Manski, CH. F. and Lerman, S. R. (1977), The Estimation of Choice Probabilities from Choice Based Samples, Econometrica, 45 (8), 1977-1988. 
McFadden, D. (1974), Conditional logit analysis of qualitative choice behaviour, in Frontiers in Econometrics (Ed.) P. Zarembka, Academic Press, New York, 105142.

Olsthoorn, X, Amann, M., Bartonova, A., Clench-Aas, J., Cofala, J., Dorland, K., Guerreiro, C., Henriksen, J.F., Jansen, H., Larssen, S. (1999), Cost Benefit Analysis of European Air Quality Targets for Sulphor Dioxide, Nitrogen Dioxide and Fine and Suspended Particulate Matter in Cities, Environmental and Resource Economics, 14, 333-351.

Ortuzar, J. de Dios and Rodríguez, G. (2002), Valuing reductions in environmental pollution in a residential location context, Transportation Research: Part D, 7, 407-427.

Röösli, M., Huss, A. and Schreier, N., Repräsentative Befragung zu Sorgen und gesundheitlichen Beschwerden im Zusammenhang mit elektromagnetischen Feldern in der Schweiz, University Bern.

Sælensminde, K. (1999), Stated choice valuation of urban traffic air pollution and noise, Transportation Research: Part D, 4, 13-27.

Shen, Junyi, Sakata, Yusuke and Hashimoto, Yoshizo (2008), Is individual environmental consciousness one of the determinants in transport mode choice?, Applied Economics, 40 (10), 1229-1239.

Siegrist, M., Earle, T. C. and Gutscher, H. (2003) Test of a trust and confidence model in the applied context of electromagnetic field (EMF) risks, Risk Analysis, 23, 705-716.

Siegrist, M., Earle, T. C., Gutscher, H. and Keller, C. (2005) Perception of Mobile Phone and Base Station Risks, Risk Analysis, 25 (5), 1253-1264.

Wardman, M. and Bristow, A. L. (2004), Traffic related noise and air quality valuations: evidence from stated preference residential choice models, Transportation Research: Part D, 9, 1-27.

\section{Acknowledgements}

The project on which this paper is based was supported by the Swiss Federal Office for the Environment (FOEN). The views expressed in this paper are those of the authors. The authors wish to thank Mehdi Farsi for his insightful and helpful 
comments and suggestions, and Daniela Pióro for her assistance with the questionnaire. Any remaining errors are responsibility of the authors. 
Table 1: Example of a choice situation

\begin{tabular}{|c|c|c|c|}
\hline SITUATION 1 & Alternative 1 & Alternative 2 & $\begin{array}{c}\text { Your current dwelling } \\
\text { situation }\end{array}$ \\
\hline Monthly rent & $1^{\prime} 774$ & $1 ' 605$ & $1^{\prime} 690$ \\
\hline View of a mobile phone antenna & No & yes & no \\
\hline $\begin{array}{l}\text { Mobile phone antenna in the } \\
\text { surrounding }(150 \mathrm{~m})\end{array}$ & $\begin{array}{l}\text { yes - with lower } \\
\text { limits }\end{array}$ & $\begin{array}{c}\text { Yes - with present } \\
\text { limits }\end{array}$ & none \\
\hline Air quality & Medium & bad & good \\
\hline Traffic noise exposure & Low & medium & medium \\
\hline My choice is: & $\frac{\circ}{0}$ & $\frac{\circ}{0}$ & $\frac{\circ}{0}$ \\
\hline
\end{tabular}


Table 2: Descriptive statistics

Socio-economic characteristics of the participants

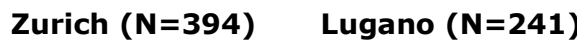

Age

Participant is a female ${ }^{a}$

40.9

40

Household income in Swiss Francs (CHF)

0.527

0.506

University education

5 '845

6'014

Household member(s) with allergy

$0.449 \quad 0.428$

$0.528 \quad 0.492$

Attributes of the current dwelling

\begin{tabular}{|c|c|c|c|}
\hline \multicolumn{2}{|l|}{ Monthly rent in $\mathrm{CHF}$} & $1 ' 585$ & $1^{\prime} 442$ \\
\hline \multicolumn{2}{|c|}{ View of a mobile phone antenna } & 0.579 & 0.531 \\
\hline \multicolumn{2}{|c|}{$\begin{array}{l}\text { Mobile phone antenna with present limit values } \\
\text { in the surrounding }(150 \mathrm{~m}) \text { : yes }\end{array}$} & 0.315 & 0.261 \\
\hline & no & 0.685 & 0.739 \\
\hline \multirow[t]{3}{*}{ Air quality: } & good & 0.223 & 0.257 \\
\hline & medium & 0.383 & 0.320 \\
\hline & bad & 0.394 & 0.423 \\
\hline \multirow[t]{3}{*}{ Traffic noise exposure: } & low & 0.363 & 0.465 \\
\hline & medium & 0.325 & 0.282 \\
\hline & High & 0.312 & 0.253 \\
\hline
\end{tabular}

\begin{tabular}{|c|c|c|}
\hline & Zurich $(N=4 ' 728)$ & Lugano( $N=2$ '884) \\
\hline Hypothetical alternatives & Sample mean & Sample mean \\
\hline Monthly rent in $\mathrm{CHF}$ & $1 ' 556$ & $1^{\prime} 410$ \\
\hline View of a mobile phone antenna* & 0.287 & 0.286 \\
\hline $\begin{array}{l}\text { Mobile phone antenna with stronger limit values } \\
\text { in the surrounding }(150 \mathrm{~m})^{* *}\end{array}$ & 0.401 & 0.406 \\
\hline No mobile phone antenna in the surrounding $(150 \mathrm{~m})^{* *}$ & 0.172 & 0.172 \\
\hline Good air quality*** & 0.335 & 0.342 \\
\hline Medium air quality*** & 0.334 & 0.333 \\
\hline Low traffic noise exposure**** & 0.327 & 0.326 \\
\hline Medium traffic noise exposure**** & 0.272 & 0.275 \\
\hline
\end{tabular}


Table 3: Estimation results of the conditional logit model for Zurich

\begin{tabular}{|c|c|c|c|c|}
\hline \multirow{2}{*}{ Variables } & \multicolumn{2}{|c|}{ Model1 } & \multicolumn{2}{|c|}{ Model2 } \\
\hline & Coeff. & Rob. t-stat. & Coeff. & Rob. t-stat. \\
\hline Status quo (constant) & $1.247 * * *$ & 15,08 & $1.196 * * *$ & 9,96 \\
\hline View of a mobile phone antenna & $-0.201 * * *$ & -2.82 & $-0.252 * * *$ & -3.29 \\
\hline $\begin{array}{l}\text { No mobile phone antenna in the surrounding } \\
(150 \mathrm{~m})\end{array}$ & $0.192 * * *$ & 2,31 & $0.322 * * *$ & 3.00 \\
\hline $\begin{array}{l}\text { Mobile phone antenna with stronger limit values } \\
\text { in the surrounding }(150 \mathrm{~m})\end{array}$ & $0.330 * * *$ & 3,98 & $0.357 * * *$ & 4,03 \\
\hline Good air quality & $1.943 * * *$ & 21.97 & $1.812 * * *$ & 14.85 \\
\hline Medium air quality & $1.266 * * *$ & 16,80 & $1.171 * * *$ & 10,49 \\
\hline Low traffic noise exposure & $2.113^{* * *}$ & 22.31 & $2.199 * * *$ & 20.62 \\
\hline Medium traffic noise exposure & $1.534 * * *$ & 17.59 & $1.592 * * *$ & 16.43 \\
\hline Monthly rent (in CHF) & $-0.003 * * *$ & -7.98 & $-0.009 * * *$ & -6.26 \\
\hline $\begin{array}{l}\text { Monthly rent } * \text { low household income (between } \\
0 \text { and } 4 \text { '000 CHF) }\end{array}$ & & & 0.0000003 & 0.57 \\
\hline $\begin{array}{l}\text { Monthly rent } * \text { high household income (5'000 } \\
\text { CHF and more) }\end{array}$ & & & $0.0000005 * * *$ & 4,02 \\
\hline Monthly rent $*$ frequency of choosing status quo & & & $0.0008 * * *$ & 4,78 \\
\hline Negative difference in the monthly rent & & & 0.0002 & 0.14 \\
\hline Bad air quality $*$ allergies & & & $-0.384 * * *$ & -2.53 \\
\hline $\begin{array}{l}\text { No mobile phone antenna in the surrounding } \\
(150 \mathrm{~m}) * \text { University education }\end{array}$ & & & $-0.291 * * *$ & -2.17 \\
\hline No. of participants & 394 & & 344 & \\
\hline No. of observations & 2634 & & 2064 & \\
\hline Log likelihood & -1741 & & -1490 & \\
\hline PseudorR2 & 0,329 & & 0,343 & \\
\hline
\end{tabular}


Table 4: Estimation results of the conditional logit model for Lugano

\begin{tabular}{|c|c|c|c|c|}
\hline \multirow{2}{*}{ Variables } & \multicolumn{2}{|c|}{ Model1 } & \multicolumn{2}{|c|}{ Model2 } \\
\hline & Coeff. & Rob. t-stat. & Coeff. & Rob. t-stat. \\
\hline Status quo (constant) & $0.890 * * *$ & 8,57 & $0.563 * * *$ & 3,53 \\
\hline View of a mobile phone antenna & $-0.339 * * *$ & -3.61 & $-0.370 * * *$ & -3.40 \\
\hline $\begin{array}{l}\text { No mobile phone antenna in the surrounding } \\
(150 \mathrm{~m})\end{array}$ & $0.458 * * *$ & 4,40 & $0.585 * * *$ & 4,22 \\
\hline $\begin{array}{l}\text { Mobile phone antenna with stronger limit values in } \\
\text { the surrounding }(150 \mathrm{~m})\end{array}$ & $0.385 * * *$ & 3,72 & $0.425 * * *$ & 3,59 \\
\hline Good air quality & $1.993 * * *$ & 16.22 & $1.721 * * *$ & 10,17 \\
\hline Medium air quality & $1.235 * * *$ & 11,72 & $1.068 * * *$ & 6,80 \\
\hline Low traffic noise exposure & $1.786 * * *$ & 16.55 & $1.909 * * *$ & 15.39 \\
\hline Medium traffic noise exposure & $1.192 * * *$ & 10,59 & $1.248 * * *$ & 9,45 \\
\hline Monthly rent (in CHF) & $-0.003 * * *$ & -5.48 & $-0.011 * * *$ & -5.20 \\
\hline $\begin{array}{l}\text { Monthly rent * low household income (between } 0 \\
\text { and } 4{ }^{\prime} 000 \mathrm{CHF} \text { ) }\end{array}$ & & & 0.000001 & 1,67 \\
\hline $\begin{array}{l}\text { Monthly rent } * \text { high household income }\left(5^{\prime} 000 \mathrm{CHF}\right. \\
\text { and more) }\end{array}$ & & & 0.0000003 & 1,66 \\
\hline Monthly rent $*$ frequency of choosing status quo & & & 0.001 & 3,51 \\
\hline Negative difference in the monthly rent & & & 0.007 & 2,76 \\
\hline Bad air quality $*$ allergies & & & $-0.539 * * *$ & -2.57 \\
\hline $\begin{array}{l}\text { No mobile phone antenna in the surrounding } \\
(150 \mathrm{~m}) * \text { University education }\end{array}$ & & & -0.081 & -0.47 \\
\hline No. of participants & 241 & & 192 & \\
\hline No. of observations & 1442 & & 1149 & \\
\hline Log likelihood & -1125 & & -864 & \\
\hline PseudorR2 & 0,289 & & 0,315 & \\
\hline
\end{tabular}

Table 5: Average Willingness to pay in CHF/month for Zurich and Lugano ${ }^{24}$

\begin{tabular}{|c|c|c|c|c|c|c|}
\hline \multirow{3}{*}{$\begin{array}{l}\text { Attribute } \\
\text { View of a mobile phone antenna }\end{array}$} & \multicolumn{3}{|c|}{ Zurich } & \multicolumn{3}{|c|}{ Lugano } \\
\hline & \multirow{2}{*}{$\begin{array}{l}\text { WTP } \\
-28\end{array}$} & \multicolumn{2}{|c|}{$\begin{array}{l}\text { Sign. } 95 \%- \\
\text { Interval }\end{array}$} & \multirow{2}{*}{$\begin{array}{l}\text { WTP } \\
-32\end{array}$} & \multicolumn{2}{|c|}{$\begin{array}{l}\text { Sign. } 95 \%- \\
\text { Interval }\end{array}$} \\
\hline & & -9 & -47 & & -10 & -55 \\
\hline \multicolumn{7}{|c|}{$\begin{array}{l}\text { Mobile phone antenna with present limit } \\
\text { values in the surrounding }(150 \mathrm{~m}) \text { : }\end{array}$} \\
\hline to no mobile phone antenna & 35 & 11 & 60 & 51 & 22 & 81 \\
\hline $\begin{array}{l}\text { to mobile phone antenna with } \\
\text { stronger limit values }\end{array}$ & 39 & 15 & 63 & 37 & 12 & 63 \\
\hline \multicolumn{7}{|l|}{ Air quality: } \\
\hline From bad to good & 198 & 133 & 263 & 151 & 88 & 214 \\
\hline From bad to medium & 128 & 86 & 171 & 94 & 54 & 133 \\
\hline From medium to good & 70 & 47 & 92 & 57 & 34 & 81 \\
\hline \multicolumn{7}{|l|}{ Traffic noise exposure: } \\
\hline From high to low & 241 & 166 & 315 & 168 & 104 & 231 \\
\hline From high to medium & 174 & 121 & 228 & 109 & 66 & 153 \\
\hline From medium to low & 67 & 45 & 87 & 59 & 38 & 78 \\
\hline
\end{tabular}

$241 \mathrm{CHF} \approx 0.66 \mathrm{EUR}(04.11 .2008)$ 